\title{
Télescope
}

Revue d'analyse comparée en administration publique

\section{L'innovation dans les services publics : gouvernance plutôt que gestion des risques}

\section{Stephen P. Osborne et Louise Brown}

Volume 19, numéro 2, printemps 2013

L'innovation dans le secteur public : au-delà des discours

URI : https://id.erudit.org/iderudit/1023842ar

DOI : https://doi.org/10.7202/1023842ar

Aller au sommaire du numéro

Éditeur(s)

L'Observatoire de l'administration publique

ISSN

1929-3348 (numérique)

Découvrir la revue

Citer cet article

Osborne, S. P. \& Brown, L. (2013). L'innovation dans les services publics : gouvernance plutôt que gestion des risques. Télescope, 19(2), 87-96. https://doi.org/10.7202/1023842ar

\section{Résumé de l'article}

Cet article étudie l'importante question des risques engendrés par les processus d'innovation dans les services publics. On y propose l'idée que les politiques publiques actuelles offrent peu d'orientation aux gestionnaires de services sur la façon de prendre en compte de tels risques, se résumant à mentionner que "c'est important ». Lorsqu'elles existent, les approches de prise en charge des risques et de l'innovation dans les services publics sont invariablement focalisées sur des optiques actuarielles ou concernées par les questions de santé et de sécurité, et ont pour objectif de minimiser ou d'éliminer les risques. Or les risques sont inhérents aux processus d'innovation et il est essentiel d'adopter une nouvelle approche qui reconnaît la nécessité des risques dans les innovations efficaces et qui s'engage de manière plus globale avec les intervenants afin de déterminer les niveaux de risque acceptables comparativement aux bienfaits potentiels pour les services publics d’une innovation donnée. Un modèle en cinq étapes est proposé pour mettre en oeuvre cette approche. 


\title{
L'INNOVATION DANS LES SERVICES PUBLICS : GOUVERNANCE PLUTÔT QUE GESTION DES RISQUES ${ }^{1}$
}

\author{
Par Stephen P. Osborne, Professeur, University of Edinburgh Business School, Écosse, \\ Royaume-Uni \\ stephen.osborne@ed.ac.uk \\ Et Louise Brown, Docteur, Département des sciences sociales et politiques, University \\ of Bath, Angleterre, Royaume-Uni \\ l.brown@bath.ac.uk \\ Traduit de l'anglais
}

RÉSUMÉ Cet article étudie l'importante question des risques engendrés par les processus d'innovation dans les services publics. On y propose l'idée que les politiques publiques actuelles offrent peu d'orientation aux gestionnaires de services sur la façon de prendre en compte de tels risques, se résumant à mentionner que "c'est important ». Lorsqu'elles existent, les approches de prise en charge des risques et de l'innovation dans les services publics sont invariablement focalisées sur des optiques actuarielles ou concernées par les questions de santé et de sécurité, et ont pour objectif de minimiser ou d'éliminer les risques. Or les risques sont inhérents aux processus d'innovation et il est essentiel d'adopter une nouvelle approche qui reconnaît la nécessité des risques dans les innovations efficaces et qui s'engage de manière plus globale avec les intervenants afin de déterminer les niveaux de risque acceptables comparativement aux bienfaits potentiels pour les services publics d'une innovation donnée. Un modèle en cinq étapes est proposé pour mettre en œuvre cette approche.

ABSTRACT This paper considers the important issue of risk in the innovation process in public services. It argues that current public policy gives little guidance to service manager son how to deal with such risk, beyond noting that 'it is important'. Where they do exist, current approaches to risk and innovation in public services are invariably around actuarial or health and safety approaches concerned with minimising or removing risk. However we argue that risk is integral to the innovation process and that an alternative approach is needed that both recognises the necessity of risk for effective innovation and engages most broadly with service stakeholders to resolve how much risk is acceptable for identified potential public service benefits. A five stage model is proposed to implement this approach.

1 Cet article est basé sur un document publié dans Public Money \& Management (vol. 31, nº 1, 2011, p. 4-6). Cet argument est également développé de manière plus approfondie dans Brown et Osborne (2013). 
$\mathrm{Au}$ Royaume-Uni, la tendance privilégiant l'innovation en tant que moyen d'améliorer l'efficacité et le rendement des services publics progresse de manière constante. NESTA employait d'ailleurs récemment l'expression «impératif d'innovation » (Harris et Albury, 2009) et le gouvernement du Royaume-Uni a publié un livre blanc intitulé Innovation Nation (Department of Innovation, Universities and Skills, 2008). La volonté de recourir à l'innovation pour réformer les services publics est grande. Cet engagement ne fait que s'affirmer à mesure que les effets de la récession sur les dépenses publiques se révèlent (Patterson et autres, 2009, p. 12). Cependant, les mêmes études ont également permis de détecter que ce climat économique peut entraîner une focalisation sur des types d'innovation moins risqués, nonobstant les besoins.

Dans le contexte de cette politique, nous sommes d'avis que la question du rôle du risque dans l'innovation, jusqu'ici négligée, mérite plus d'attention (Brown, 2010; Osborne, 1998; Osborne et Brown, 2011) ${ }^{2}$. Dans un contexte de service public, le risque est souvent présenté comme un phénomène négatif, au mieux comme une chose à minimiser, sinon à éviter. Pourtant, les auteurs traitant de l'innovation tant dans les secteurs privé que public ont souligné l'importance du risque dans le succès de l'innovation (Borins, 2001; Singh, 1986). Nous estimons que le cadre actuel du service public ne facilite pas la gestion efficace des risques au sein des processus d'innovation et prenons parti pour une solution basée sur la gouvernance de risques négociée, plutôt que pour la minimisation.

\section{LA GESTION DE L'INNOVATION ET DU RISQUE DANS UN CONTEXTE DE SERVICE PUBLIC}

Dans l'ensemble de la littérature sur la gestion, la plupart des textes portant sur la gestion des risques proviennent soit du secteur financier et des entreprises (Stulz, 1996), soit de l'industrie de la santé et de la sécurité (Rasmussen, 1997). Dans ces deux cas, les définitions du risque sont décrites en termes péjoratifs : "le risque est la probabilité que se produise un événement défavorable particulier au cours d'une période de temps déterminée... la probabilité de quelque événement négatif... » (Breakwell, 2007, p. 2). Un autre thème récurrent dans la documentation est la distinction entre "risque » et "incertitude », le risque étant la prise de décision dans un contexte où les options sont connues et leurs résultats probables, alors que l'incertitude fait référence à la prise de décision dans un contexte où les options ainsi que leurs éventuels résultats sont inconnus (Tversky et Fox, 1995).

S'agissant des services publics, la politique en place au Royaume-Uni offre un guide sur la gestion de risque (HM Treasury, 2004; National Audit Office, 2004), mais celui-ci ne fait pas référence à la nature souvent controversée des services publics et à leurs bienfaits, ni aux défis représentés par la gestion des risques et des bienfaits potentiels dans les processus d'innovation. L'Audit Commission a également admis l'importance du risque dans le processus d'innovation, mais encore une fois, elle offre peu de conseils, hors les discours selon lesquels la gestion des risques est « d'importance capitale pour s'assurer que les projets innovants gardent le cap » et que les autorités croient qu'elle "contribue de manière considérable à la réalisation de projets innovants » (Audit Commission, 2007, p. 40). De façon similaire, le livre blanc intitulé Innovation Nation signalait que les politiques publiques actuelles pourraient engendrer une " aversion accrue au risque » dans les services publics

2 Il est révélateur qu'une publication sur le risque et les services publics de ESRC (Hood et Miller, 2009), au demeurant excellente, ne fait nulle mention des risques liés à l'innovation dans les services publics. 
(Department of Innovation, Universities and Skills, 2008, p. 72), mais sans formuler de conseils précis pour corriger cette situation. La Public Management Risk Association (ALARM) reconnaît les risques controversés dans les services publics, mais encore une fois adopte une approche implicite de minimisation des risques, sous prétexte que les risques font porter un fardeau financier inutile aux services publics (Williams, 2009). En Écosse, le nouveau cadre stratégique pour l'innovation (Scottish Government, 2009), de manière inquiétante, ne fait aucune mention de la gestion des risques relatifs à l'innovation dans les services publics.

Au sein des comptes rendus de recherche sur les services publics, il existe également une mince et insatisfaisante documentation sur le risque et la gestion des risques. Par exemple, Hood (2002) prétend que la gestion des risques est plus concernée par la distribution des blâmes que par la sécurité, Vincent (1996) soutient que l'étude minutieuse des services publics rend l'innovation plus difficile que dans le secteur privé, alors que Harman (1994) affirme que l'innovation a en vérité diminué l'imputabilité des services publics et ouvert la voie à une augmentation des cas de fraude.

Il est également clair, selon l'analyse de Vincent, qu'une grande part de la littérature du secteur public est concernée par la responsabilité personnelle ou organisationnelle (assurable) pour les décisions de services au sein d'un État décentralisé, plutôt que par les cas plus spécifiques de risque dans les décisions liées aux services. Lorsque la seconde proposition fait l'objet d'une exploration, c'est invariablement au sein d'un contexte professionnel, tel que dans les services sociaux (Munro, 2009) ou la recherche (Leung et Isaacs, 2008), plutôt que dans un contexte public et social. Les politiques sociales et publiques ne se donnent la peine d'explorer la question des risques, conclut Vincent en ramenant la discussion vers Hood, que lorsque les organisations publiques cherchent à se protéger de l'étude minutieuse du public et de la reddition de comptes. La capacité de ces documents à offrir des conseils sensés ou à aider la compréhension de l'innovation dans les services publics est, selon nous, limitée à l'extrême. En effet, un ouvrage plutôt ambitieux portant sur la gestion des risques ne fait que trois vagues références au risque et à l'innovation dans les services publics, et toutes signifient que « l'innovation engendre un certain degré de risque » (Drennan et McConnell, 2007, p. 43).

La documentation plus générale portant sur la gestion des risques est également problématique dans le cas des services publics en raison de la contestabilité. L'approche choisie est souvent « technocratique », basée sur les évaluations " objectives » des risques selon des bases scientifiques (Pender, 2001). Alors que cette vision peut s'avérer très appropriée pour certains types d'innovation, elle ne l'est pas pour d'autres domaines où la nature des risques et leurs bénéfices potentiels sont controversés, ce qui est souvent le cas pour les innovations dans de nombreux domaines des services publics. Une autre approche est de type décisionnel. Celle-ci va au-delà de la pure domination professionnelle dans le processus d'évaluation des risques pour y incorporer l'engagement politique, mais elle ne va pas jusqu'à s'engager avec tous les intervenants, dont les usagers des services et la communauté élargie.

Il existe une approche qui va plus loin, celle de Renn (2008). Ce dernier prétend que le risque et les niveaux de risque acceptables sont des phénomènes socialement construits. Ainsi la gestion des risques requiert non seulement l'application de connaissances scientifiques, mais également un processus politique (au sens large) de négociation. Les valeurs sociales doivent être intégrées aux indices de niveaux de risque et aux résultats. Ainsi, la «gouvernance des risques » (par opposition à la gestion des risques) nécessite un processus 
inclusif centré sur les vastes débats politiques portant sur les niveaux de risque acceptables pour des innovations précises, des négociations interpersonnelles et interorganisationnelles, ainsi qu'un engagement envers les communications en cours sur les risques et une participation complète dans leur gouvernance. La question au cœur de ces processus, selon Renn, n'est pas "quel est le niveau de sécurité sous-entendu par le mot sécuritaire », mais plutôt « quel niveau d'incertitude et d'ignorance (à propos des risques véritables ou potentiels) les principaux intervenants sont-ils prêts à accepter en échange d'un bienfait donné » (Renn, 2008, p. 277).

Une telle approche négociée de la gestion des risques peut offrir beaucoup aux politiques dans le secteur public. Elle mettrait à nu la nature controversée des innovations et de leurs résultats dans les services publics et fournirait un cadre pour négocier les niveaux de risque inhérents à ces innovations en termes de société, d'organisations, d'usagers des services, de professionnels et d'employés. Cette méthode met l'accent sur la communication et la participation, en rappelant que de telles innovations dans les services viennent précisément de la collaboration entre les usagers et les fournisseurs de services (Alam, 2006). Ainsi, un cadre pour la gouvernance des risques en innovation dans les services publics est certainement un préalable essentiel pour l'avenir et représente un pas en avant par rapport aux approches mécanistes et technocratiques de la gestion des risques actuellement en vigueur dans le secteur des politiques publiques.

\section{LA GOUVERNANCE DES RISQUES POUR L'INNOVATION DANS LES SERVICES PUBLICS}

Brown et Osborne (2013) ont poussé cet argument plus loin. Ils ont élaboré un cadre général qui relie la nature du processus d'innovation au type de gouvernance des risques approprié. Ils ont également imaginé la forme que pourrait prendre un tel processus de gouvernance des risques pour l'innovation dans les services publics. Ils proposent un processus de mise en œuvre en cinq étapes pour la gouvernance des risques. Ces étapes sont présentées ici de manière plutôt linéaire, bien qu'elles fassent, en réalité, invariablement l'objet de chevauchements. On pourrait les qualifier d'itératives et on ne peut aisément les isoler les unes des autres.

Étape 1

L'identification de l'innovation
La première étape consiste à déterminer le type d'innovation à implanter et à adopter un mode de gouvernance des risques approprié, adapté à la nature et au type de l'innovation. Des modes de gouvernance des risques différents seront nécessaires pour les différents types d'innovations et les différents types de risques. Nous n'établissons pas de distinction normative entre ces modes d'analyse du risque - la négociation des risques n'est pas nécessairement "meilleure » que la gestion technocratique des risques. Comme cela deviendra apparent à mesure que la mise en œuvre de ce cadre sera explorée, différentes innovations dans les services publics nécessitent différents modes de gouvernance des risques et de la transparence. La clé est donc d'établir un mode approprié d'évaluation des risques pour chaque catégorie d'innovation et de créer une procédure claire pour chacun d'eux. 
Étape 2

L'identification

du risque

Étape 3

La collecte de

l'information
La deuxième étape consiste à se montrer explicite quant aux types de risques et à leur aire d'influence. Selon Brown et Osborne (2013), les risques de l'innovation se situent dans trois domaines. Ils peuvent être individuels, impliquant un risque direct pour les usagers du service (par exemple une approche innovante de la chirurgie cérébrale); organisationnels, impliquant des risques pour la durabilité ou la légitimité des organisations de service public ou pour le personnel professionnel impliqué dans l'innovation (par exemple les conférences familiales - Family Group Conferencing); comportementaux, impliquant des risques pour la communauté en général et pour les intervenants (par exemple les risques engendrés par le traitement dans la communauté de personnes atteintes d'importants et dangereux troubles mentaux). La clarification du lieu des risques est vitale dans la sélection d'une approche de gouvernance.

La troisième étape consiste à rassembler toute l'information disponible sur les risques et les bénéfices potentiels ou perçus des résultats de l'innovation. Une fois identifiés la nature et les caractéristiques de l'innovation, le mode approprié de gouvernance et le type de risques impliqués, cette étape est consacrée à la collecte d'information et de données empiriques, puis aux liens avec les possibles résultats. Alors que le projet va de l'avant vers une approche de la gouvernance des risques moins technocratique et moins décisionnelle, une acceptation des différents types et niveaux de données empiriques est nécessaire, de même que la reconnaissance du degré de qualité de ces preuves et de leurs limites. Dans certains domaines de développement des services faisant réellement preuve d'innovations révolutionnaires, la banque de preuves et de données peut se révéler non pas basée sur la recherche, mais sur ce qu'on appelle les «règles de l'art » ou les «pratiques exemplaires» (Slowinski et Sagal, 2010). En mesurant les données (ou leur absence), il faudra avoir recours au jugement professionnel quant aux probables résultats, ainsi qu'à une réflexion sur toute expérimentation spécifique ou tout projet pilote innovateur qui pourrait soutenir le processus. Il serait important que les organismes de service public cherchent comment l'expérimentation peut être employée afin de favoriser l'information du niveau de risque que présente une innovation (en tant qu'aide, et non comme substitut du grand processus de prise de décision).

Pour les innovations nécessitant uniquement une gouvernance des risques de type technocratique, il se pourrait bien que le processus se termine ici. Pour celles exigeant une gouvernance des risques décisionnelle ou négociée, deux autres étapes sont requises. 
Étape 4

La mise en place

d'une

collaboration
La quatrième étape consiste à établir un processus de collaboration qui permet d'instaurer une négociation avec l'ensemble des intervenants afin d'atteindre une compréhension commune des niveaux de risque acceptables, y compris la reconnaissance des visions contradictoires ainsi qu'un procédé pour tenter de les résoudre. La gouvernance décisionnelle limitera la collaboration aux partenaires organisationnels et politiques, alors que la gouvernance négociée des risques nécessitera une plus vaste inclusion. Cette étape exige également l'élaboration d'une stratégie claire qui comprend les changements requis aux comportements organisationnels et individuels. Il s'agit d'un des aspects les plus importants et potentiellement difficiles du cadre intégré, puisque celui-ci reconnaît le besoin de la négociation en vue de la construction de ce qui constitue un risque, de son lieu et de l'équilibre entre les risques et les bénéfices (potentiels) offerts par l'innovation. Par leur nature, tous les bienfaits d'une innovation dans les services publics sont potentiels, et souvent contestés. En plus des risques associés à une innovation, il existe également des risques de deuxième degré : risque qu'une innovation échoue, qu'elle ne livre pas les bienfaits espérés ou qu'elle entraîne des conséquences involontaires qui soient plus importantes que ses bienfaits.

Sans cette étape, la nature des risques plus complexes peut demeurer obscure et menaçante, augmentant ainsi les probabilités de résistance à une innovation en raison des peurs et des opinions erronées (Kinder, 2000). Les intervenants doivent s'engager dans un débat ouvert sur la nature et le type de risques impliqués par une innovation, en plus des attentes et des bienfaits potentiels. Au cours du processus de participation, la mise sur pied d'un forum de collaboration et d'un processus de consultation comprenant tous les intervenants, public inclus, et au cours duquel les risques seront débattus, sera d'une importance critique. On devra y trouver une information et une compréhension partagées en ce qui a trait aux principaux risques et incertitudes à explorer, en plus des questions de droit, de choix et de contrôle. Les risques devront être clairement définis et débattus publiquement, pesés en fonction de leurs caractéristiques relatives, de leurs coûts, de leurs bienfaits potentiels, jusqu'à ce qu'une compréhension des risques partagés et acceptables soit obtenue. Pour chaque intervenant, les risques impliqués devront être déterminés, que ce soit sur le plan de la réputation ou sur le plan personnel, politique ou même financier. Ces questions seront abordées ci-dessous.

Dans cette approche, comme l'ont démontré Reay et Hinings (2010), les activités de collaboration impliquant des intervenants dotés d'identités et d'intérêts variés sont inévitablement complexes et stressantes, même lorsque les parties ont convenu de tenter d'obtenir des résultats partagés. Ces auteurs suggèrent que des rivalités peuvent exister entre les intervenants, en particulier lorsque certains d'entre eux tiennent au maintien du statu quo. Ce 
problème pourrait facilement éclater au cœur d'un réseau d'intervenants rassemblés pour débattre d'un programme d'innovation ou d'un service public et de ses risques potentiels. L'innovation pourrait menacer les relations de pouvoir existant au sein du réseau ou encore privilégier certains intervenants au détriment d'autres (Pettigrew, 1973). Il ne serait donc pas inusité que la nature d'un risque soit contestée ou qu'elle fasse l'objet de visions opposées, entraînant des résistances de la part de l'un ou de plusieurs intervenants. Reay et Hinings croient que si les intervenants parviennent à mettre en place des mécanismes de collaboration qui permettent à des points de vue opposés de coexister, les changements deviennent possibles, bien que des divergences puissent demeurer vives pendant longtemps.

Cela peut constituer un défi, en particulier dans un contexte de service public dans lequel les considérations et les intérêts politiques à court terme peuvent entrer en conflit avec les besoins d'innovation à long terme sur le plan des politiques ou des services. Malgré qu'il s'agisse sans nul doute d'une tâche difficile, poursuivre sans une telle approche sophistiquée a plus de chance d'entraîner des échecs ou des insatisfactions dans les processus d'innovation. Heureusement, les outils qui permettent de piloter ce processus exigeant dans un contexte de service public existent déjà. Crosby et Bryson (2005) et Bryson (2004) offrent un tel modèle, basé sur l'analyse des intervenants. Ils ciblent la manière par laquelle un gouvernement peut soutenir un pareil processus en fournissant des cadres dynamiques ou législatifs, des ressources et des groupes de multiples secteurs. Selon eux, quels que soient les intervenants dans un contexte politique donné, le processus de changement doit être géré par la collaboration et non par décret. Pour que l'entreprise soit couronnée de succès, les partenaires de la collaboration doivent développer un sens commun des résultats désirés, un processus clairement établi qui fait la part belle au leadership, à la confiance, à la bonne planification, à la gestion des conflits et au partage de l'information et de la connaissance.

Certes, cette étape engendre des coûts en temps et en argent. Elle ne garantit pas non plus le succès. La négociation de points de vue contradictoires est un processus inévitablement stressant. Cependant, pour les innovations plus complexes qui nécessitent des types de gouvernance décisionnelle ou caractérisée par des risques transparents, il est essentiel d'engager la plus vaste communauté d'intervenants possible dans la négociation des niveaux de risque jugés acceptables en rapport avec les bienfaits potentiels. Il est tout à fait possible que des voix dissidentes minoritaires s'opposent à la voie choisie au final, mais la reconnaissance de ces voix les rendra plus à même de se ranger à la résolution ou à l'accommodation que si elles sont ignorées ou rejetées. Ainsi, un cadre de gouvernance des risques qui permet à des organisations 


\title{
Étape 5 Un système de reddition de comptes
}

de reconnaître, d'accepter et de gérer les risques, et non seulement de les minimiser, nécessitera l'incorporation d'étapes clairement définies faciles à suivre pour toutes les parties prenantes. Le processus devrait comprendre des "voies d'évasion » pour les professionnels ou les usagers des services qui estimeraient que les risques sont trop importants pour eux, sans pour autant que cela ne porte atteinte à l'innovation.

Finalement, la cinquième étape est la mise sur pied nécessaire d'un système de reddition de comptes au sein du processus. Il convient de s'entendre à l'avance avec les intervenants sur les résultats et d'établir un mécanisme d'évaluation formative et sommative. Des révisions fréquentes ainsi que des rétroactions peuvent allouer aux personnes impliquées un niveau de contrôle sur le processus d'innovation et sur les risques que le projet représente pour eux. Selon Crosby et Bryson :

\begin{abstract}
Des études quantitatives sur la progression de divers indicateurs peuvent être combinées à des méthodes qualitatives, comme les groupes de discussion, les observations des participants et les entrevues, de manière à découvrir ce qui constitue un succès pour différentes clientèles et ce qui contribue à l'accomplissement des objectifs de la collaboration. Les questions devraient être pertinentes pour chaque groupe. (Crosby et Bryson, 2005, p. 16)
\end{abstract}

\section{CONCLUSION}

Cet article démontre l'importance grandissante de l'innovation ainsi que l'intérêt qu'elle suscite en tant que moyen d'améliorer l'efficacité des services du secteur public. Les structures actuelles des services publics ne permettent pas toujours, ni ne facilitent, la gestion efficace des risques au cœur du processus d'innovation. Le présent document propose une approche basée sur la gouvernance des risques négociée plutôt qu'une approche fondée sur leur minimisation. En passant en revue la documentation consacrée à la gestion des risques, on note que le risque est souvent perçu de manière négative, ce qui entraîne une conception et une gestion des risques très particulières. Au Royaume-Uni, les approches politiques existantes liées à la gestion des risques sont avares de conseils, suggérant au mieux de les réduire au maximum, au pire de les ignorer entièrement. En privilégiant un processus plus à même de reconnaître et de soutenir les questions liées à la gestion des risques et à l'innovation dans le contexte des services publics, cet article présente un cadre en cinq étapes de mise en place d'un processus de gestion des risques. Le cadre en question nécessite de la part des organisations qu'elles définissent explicitement les risques potentiels et les bénéfices escomptés auprès des réseaux d'intervenants inévitablement impliqués. Il est ainsi recommandé d'établir des formes de collaboration favorisant une négociation transparente des risques et des bénéfices. Cette approche représente un défi, mais l'instauration de tels processus permet une surveillance constante des risques et un réel engagement dans l'innovation des services publics, tout en garantissant une gestion des risques négociée. 


\section{BIBLIOGRAPHIE}

Alam, I. (2006). « Removing the Fuzziness from the Front-end of Service Innovations through Customer Interactions », Industrial Marketing Management, vol. 35, n 4, p. 468-480.

Audit Commission (2007). Seeing the Light: Innovation in Local Public Services, Londres, Audit Commission.

Breakwell, G. M. (2007). The Psychology of Risk, Cambridge, Cambridge University Press.

Borins, S. (2001). The Challenge of Innovating in Government, Washington DC, IBM Center for the Business of Government.

Brown, L. (2010). «Balancing Risk and Innovation to Improve Social Work Practice », British Journal of Social Work, vol. 40, no 4, p. 1211-1228.

Brown, L. et S. Osborne (2013). «Risk and Innovation: Towards a Framework for Risk Governance in Public Services », Public Management Review, vol. 15, no 2, p. 186-208.

Department of Innovation, Universities and Skills (2008). Innovation Nation, Londres, Department of Innovation, Universities and Skills.

Bryson, J. (2004). «What to Do When Stakeholders Matter », Public Management Review, vol. 6, $\mathrm{n}^{\circ} 1$, p. 21-53

Crosby, B. et J. Bryson (2005). Leadership for the Common Good, San Francisco, Jossey Bass.

Drennan, L. et A. McConnell (2007). Risk and Crisis Management in the Public Sector, Londres, Routledge.

H M Treasury (2004). The Risk Programme. Improving Government's Risk Handling, Final Report to the Prime Minister, Londres, HM Treasury.

Harman, E. (1994). "Accountability and Challenges for Australian Governments », Australian Journal of Political Science, vol. 29, no 1, p. 1-17.

Harris, M. et D. Albury (2009). The Innovation Imperative, Londres, NESTA.

Hood, C. (2002). «The Risk Game and the Blame Game», Government and Opposition, vol. 37, no 1 , p. 15-37.

Hood, C. et P. Miller (2009). Risk and Public Services, Oxford, ESRC Public Services Programme.

Kinder, T. (2000). «A sociotechnical approach to the innovation of a network technology in the public sector », European Journal of Innovation Management, vol. 3, n 2, p. 72-90.

Leung, F. et F. Isaacs (2008). «Risk Management in Public Sector Research: Approach and Lessons Learned at a National Research Organization », $R \& D$ Management, vol. 38, $\mathrm{n}^{\circ}$ 5, p. 510-519.

Munro, E. (2009). « Managing Societal and Institutional Risk in Child Protection », Risk Analysis, vol. 29, no 7, p. 1015-1023.

National Audit Office (2004). Managing Risks to Improve Public Services, Londres, NAO.

Osborne, S. (1998) « The Innovative Capacity of Voluntary Organizations: Implications for Local Government », Local Government Studies, vol. 24, no 1, p. 19-40.

Osborne, S. et L. Brown (2011). «Innovation, Public Policy and Public Services Delivery in the UK: The Word That Would Be King? », Public Administration, vol. 89, nº 4, p. 1335-1350. 
Patterson, F. et autres (2009). Everyday Innovation: How to Enhance Innovative working in employees and Organisations, Londres, NESTA.

Pender, S. (2001). « Managing Incomplete Knowledge: Why Risk Management is not Sufficient », International Journal of Project Management, vol. 19, no 2, p. 79-87.

Pettigrew, A. (1973). The Politics of Organizational Decision Making, Londres, Taylor \& Francis.

Pheysey, D. (1993). Organizational Cultures: Types and Transformations, Londres, Routledge.

Rasmussen, J. (1997). « Risk Management in a Dynamic Society: A Modelling Problem », Safety Science, vol. 27, no 2/3, p. 183-223.

Reay, T. et C. R. Hinings (2010). "Managing the Rivalry of Competing Institutional Logics», Organization Studies, vol. 30, nº 6, p. 629-652.

Renn, O. (2008). Risk Governance: Coping with Uncertainty in a Complex World, Londres, Earthscan.

Scottish Government (2009). Innovation for Scotland: A Strategic Framework for Innovation in Scotland, Edinburgh, Scottish Government.

Singh, J. (1986). "Performance, Slack, and Risk Taking in Organizational Decision Making», Academy of Management Journal, vol. 29, n 3, p. 562-585.

Slowinski, G. et M.W. Sagal (2010). "Good Practices in Open Innovation », Research-Technology Management, vol. 53, $\mathrm{n}^{\circ}$ 5, p. 38-45.

Stulz, R. (1996). "Rethinking Risk Management», Journal of Applied Corporate Finance, automne, p. 8-24.

Tversky, A. et R. Fox (1995). "Weighing Risk and Uncertainty », Psychological Review, vol. 102, $n^{\circ} 2$, p. 269-283.

Vincent, J. (1996). « Managing Risk in Public Services: A Review of the International Literature », International Journal of Public Sector Management, vol. 9, $\mathrm{n}^{\circ}$ 2, p. 57-64.

Williams, G. (2009). Applying Management of Risk (M_o_R) for Public Services, Sidmouth, ALARM. 